\title{
1 Lifelong single-cell profiling of cranial neural crest
}

2 diversification

3

4 Peter Fabian ${ }^{\# 1}$, Kuo-Chang Tseng" ${ }^{\# 1}$, Mathi Thiruppathy ${ }^{\# 1}$, Claire Arata ${ }^{\# 1}$, Hung-Jhen Chen ${ }^{1}$, 5 Joanna Smeeton ${ }^{1,2}$, Nellie Nelson ${ }^{1}$, and J. Gage Crump*1

6

$7 \quad{ }^{1}$ Eli and Edythe Broad California Institute for Regenerative Medicine Center for Regenerative

8 Medicine and Stem Cell Research, Department of Stem Cell Biology and Regenerative Medicine,

9 University of Southern California Keck School of Medicine, Los Angeles, CA 90033, USA

$11{ }^{2}$ Department of Rehabilitation and Regenerative Medicine, Columbia University Irving Medical

12 Center, Columbia University, New York, NY 10032, USA

13

$14 \quad$ "These authors contributed equally

15 *Correspondence: J. Gage Crump, gcrump@usc.edu, (323) 442-2693

16

17 Running Title: Single-cell profiling of neural crest

18

Keywords: Cranial neural crest; single-cell genomics; craniofacial skeleton; chromatin accessibility; zebrafish 


\section{Highlights}

\section{Graphical Abstract}

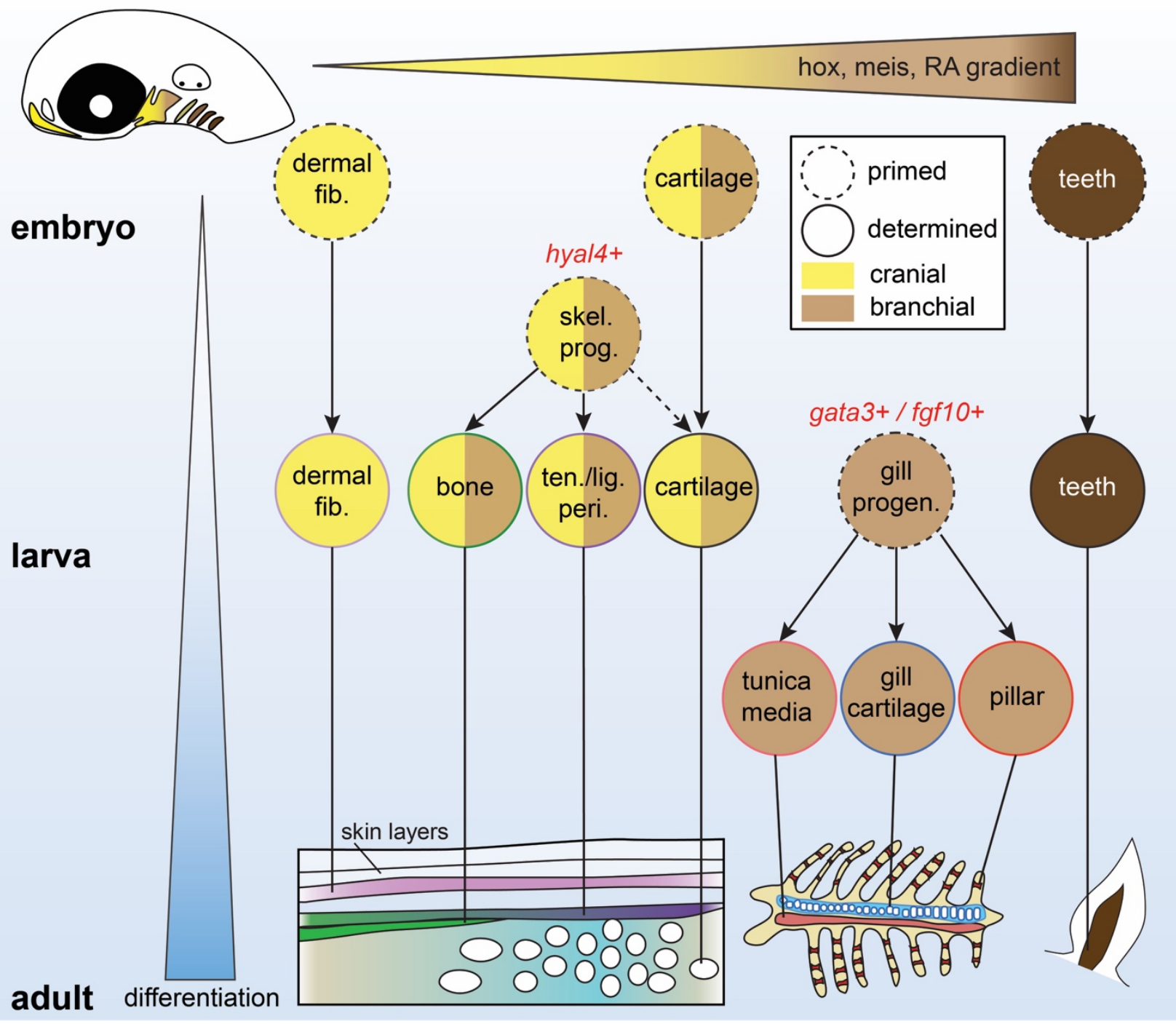




\section{Abstract}

35 The cranial neural crest generates a huge diversity of derivatives, including the bulk of connective

36 and skeletal tissues of the vertebrate head. How neural crest cells acquire such extraordinary

37 lineage potential remains unresolved. By integrating single-cell transcriptome and chromatin

38 accessibility profiles of cranial neural crest-derived cells across the zebrafish lifetime, we observe

39 region-specific establishment of enhancer accessibility for distinct fates. Neural crest-derived cells rapidly diversify into specialized progenitors, including multipotent skeletal progenitors, stromal cells with a regenerative signature, fibroblasts with a unique metabolic signature linked to skeletal integrity, and gill-specific progenitors generating cell types for respiration. By retrogradely mapping the emergence of lineage-specific chromatin accessibility, we identify a wealth of candidate lineage-priming factors, including a Gata3 regulatory circuit for respiratory cell fates.

Rather than multilineage potential being an intrinsic property of cranial neural crest, our findings support progressive and region-specific chromatin remodeling underlying acquisition of diverse neural crest lineage potential.

\section{Main text}

50 Cranial neural crest-derived cells (CNCCs) are a vertebrate-specific population, often referred to as the fourth germ layer, that have extraordinary potential to form diverse cell types. In addition to pigment cells and the peripheral nervous system, CNCCs form the ectomesenchyme that populates the pharyngeal arches and gives rise to much of the skeleton and connective tissue of the jaws and face ${ }^{1}$. Posterior arch CNCCs contribute to a distinct set of organs, including the thymus, parathyroid, and cardiac outflow tract, and in fishes cell types important for respiration, 
59 The extent to which diverse lineage potential is an intrinsic property of CNCCs, or acquired

60 through later inductive signaling, has been investigated for over a century through labeling,

61 grafting, and extirpation experiments, yet remains unresolved ${ }^{3}$. Individual avian CNCCs can

62 generate multiple types of derivatives in vitro, including ectomesenchyme and neuroglial cells,

63 suggesting multilineage potential is an intrinsic property ${ }^{4}$. However, upon cranial transplantation,

64 trunk neural crest cells, which normally do not make mesenchymal derivatives, can contribute to

65 the facial skeleton following extended culture ${ }^{5}$ or misexpression of key transcription factors ${ }^{6}$. A

66 recent study in skate also shows mesodermal contribution to the gill skeleton, a classically

67 considered CNCC-derived structure ${ }^{7}$. These findings point to extrinsic inductive cues for CNCC

68

69 fate determination. Here we take a genomics approach in zebrafish to understand when enhancers linked to diverse CNCC fates first gain accessibility, thus revealing that chromatin accessibility underlying multilineage potential is largely gained after CNCC migration.

\section{Single-cell atlas of CNCC derivatives across the zebrafish lifetime}

To understand the emergence and diversification of CNCC lineages across the lifetime of a vertebrate, we constructed a longitudinal single-cell atlas of gene expression and chromatin accessibility of zebrafish CNCC derivatives. We permanently labeled CNCCs using Sox10:Cre; actab2:IoxP-BFP-STOP-loxP-dsRed (Sox10>dsRed) fish (Fig. 1a), in which genetic recombination indelibly labels CNCCs shortly after their specification at 10 hours post-fertilization $(\mathrm{hpf})^{8}$. Previous single-cell analyses of CNCCs in zebrafish ${ }^{9}$, chick $^{10}$, and mouse ${ }^{11-13}$, and in vitro human CNCC-like cells ${ }^{14}$, had focused on CNCC establishment, migration, and early fate choices between the neuroglial, pigment, and ectomesenchyme lineages. Here we investigate cellular diversity and lineage progression of CNCC ectomesenchyme across embryonic (1.5 and 2 days post-fertilization (dpf)), larval (3 and $5 \mathrm{dpf}$ ), juvenile (14 and $60 \mathrm{dpf}$ ), and adult (150-210 dpf) stages. After fluorescence activated cell sorting (FACS) of Sox10>dsRed+ cells from dissected heads, we performed single-cell RNA sequencing (scRNAseq) and single-nuclei assay for 
transposase accessible chromatin sequencing (snATACseq) at each stage using the 10X Genomics Chromium platform and paired-end Illumina next-generating sequencing (Fig. 1b). After filtering for quality, we obtained 58,075 cells with a median of 866 genes per cell for scRNAseq, and 88,177 cells with a median of 10,449 fragments per cell for snATACseq. To better resolve snATACseq data, we used the SnapATAC package ${ }^{15}$, which integrates snATACseq with scRNAseq data to create "pseudo-multiome" datasets.

Analysis of CNCC cell clusters across all stages using UMAP dimensionality reduction recovered most known CNCC derivatives, including Schwann cells (glia), several neuronal subtypes, pigment cells, and diverse mesenchymal cell types (Fig. S1-8, Table S1). We also recovered otic placode and epithelial cells, likely reflecting additional non-CNCC expression of Sox10:Cre ${ }^{8}$, and blood lineage cells, likely due to autofluorescence. Similar clusters were recovered using both scRNAseq and SnapATAC data. We then re-clustered the CNCC ectomesenchyme subpopulation across stages, as this makes the most substantial and diverse cell contributions in the head. To confirm ectomesenchyme identity at $1.5 \mathrm{dpf}$, we also performed scRNAseq analysis of cells double-positive for the CNCC transgene sox10:dsRed and the ectomesenchyme transgene fli1a:eGFP. Co-clustering showed high concordance between sox10:dsRed+; fli1a:eGFP+ ectomesenchyme and the Sox10>dsRed+ ectomesenchyme subset, and between Sox10>dsRed+ ectomesenchyme scRNAseq subsets at all 7 stages (Fig. S8).

At the adult stage, we recovered 17 distinct clusters using scRNAseq that corresponded to 16 clusters using SnapATAC; these were largely associated with the jaw skeleton or gills (Fig. 1c-e). Skeletal derivatives include bone, cartilage, teeth, and a population with properties of periosteum, tendon, and ligament. Gills are composed of primary filaments containing cartilage rods and primary veins surrounded by a tunica media, and numerous secondary filaments housing endothelial-like "pillar" cells that promote gas exchange. Unexpectedly, we recovered a 
111 specialized type of gill cartilage distinct from that in the rest of the head, as well as pillar and

112 tunica media cells and putative gill progenitors. We also recovered smooth muscle, perivascular,

113 and stromal cells (see Table S1 for cluster marker genes and Fig. S9-10 for in situ validation).

115 In addition to skeletal and gill populations, we recovered a distinct type of fibroblast enriched for 116 the cell adhesion molecule chl1a and wnt5a. Strikingly, these fibroblasts are also enriched for 117 genes encoding enzymes for all steps of phenylalanine and tyrosine breakdown (Fig. 1f, Fig. S11).

118 In situ hybridization for two of these genes ( $h p d b$ and $p a h)$ reveals that these fibroblasts are in 119 the dermis between the skin epidermis and runx2b+/sp7+ osteoblast lineage cells (Fig. 1g,h). 120 Humans with mutations in $H G D$, which encodes an intermediate enzyme in the Phe/Tyr catabolic 121 pathway, develop Alkaptonuria, or black bone disease, due to accumulation and pathological 122 aggregation of homogentisic $\operatorname{acid}^{16}$. As the abundant melanocytes in the zebrafish skin use high 123 levels of Tyr to synthesize melanin, one possibility is that these specialized dermal fibroblasts function to protect the skeleton by removing damaging Phe/Tyr metabolites.

\section{Progressive emergence of CNCC derivatives and region-specific progenitors}

127 To understand lineage decisions of CNCC mesenchyme across time, we first used the STITCH 128 algorithm ${ }^{17}$ to connect individual stages into developmental trajectories for scRNAseq and 129 snATACseq datasets (Fig. 2a,b). As early as $3 \mathrm{dpf}$ (particularly apparent for snATACseq), we observe divergence of CNCCs into skeletogenic versus gill lineages. A hyal4+ perichondrium population precedes branches for tendon/ligament, periosteum, and osteoblasts (Fig. S9), and an fgf10b+ gill progenitor population appears at $5 \mathrm{dpf}$ and precedes branches for gill cartilage, pillar, and tunica media cells (Fig. S10). We also observe a distinct trajectory to dermal fibroblasts by 3 dpf (Fig. S11), as well as to cxcl12a+ stromal cells (Fig. S9) and teeth. We do not observe CNCC contributions to cardiomyocytes (Fig. S8), in contrast to reports for amniotes ${ }^{18}$. By creating an 
differentiation, we found no evidence for retention of ectomesenchyme identity at later stages, as shown by aggregated ectomesenchyme gene expression and the early ectomesenchyme marker $n r 2 f 5^{19}$ (Fig. S12). Although formation of CNCC ectomesenchyme involves a reacquisition of the pluripotency network ${ }^{14}$, we also did not observe expression of pluripotency genes pou5f3 (oct4), sox2, nanog, and klf4 at any stage of post-migratory ectomesenchyme, with the exception of

142 lin28aa that displays broad expression at $1.5 \mathrm{dpf}$ and is rapidly extinguished by $2 \mathrm{dpf}$ (Fig. S12).

143 Rather than maintenance of a multipotent ectomesenchyme population, our data point to progressive emergence of specialized hyal4+ perichondrium, cxc/12a+ stromal, and fgf10a/b+ gill populations at $3 \mathrm{dpf}$ and beyond (Fig. S12).

To further dissect region-specific lineages, we used Monocle $3^{20}$ on scRNAseq datasets to construct pseudotime trajectories of anterior arch (i.e. skeletogenic) versus posterior arch (i.e. gill, hoxb3a+/gata3+) CNCC mesenchyme at 5-14 dpf (Fig. S13, dermal fibroblasts and teeth were complimentary pattern to postnb and col10a1a expression in periosteum (Fig. S9). Branches from hyal4+ perichondrium led to periosteum, tendon and ligament cells, chondrocytes, and osteoblasts, consistent with studies showing perichondrium to be the precursor of the periosteum

157 in endochondral bones ${ }^{21,22}$.

159 For gill clusters, cell distribution from 5 to $14 \mathrm{dpf}$ revealed two primary trajectories (Fig. 2f-h, Fig. $160 \mathrm{~S} 13)$. In the first, $c x c / 12 a+/ c c / 25 b+$ stromal cells give rise to mesenchyme associated with retinoic 161 acid metabolism (aldh1a2+/rdh10a+), with in situ hybridization revealing these cell types restricted 162 to the base of secondary filaments (Fig. S10). In the second, fgf10a+ cells are connected to 
fgf10b+ cells, which then diverge into gill cartilage, pillar, tunica media, and perivascular populations. To test whether fgf10b+ cells are progenitors for specialized gill subtypes, we used CRISPR/Cas9 to insert a photoconvertible nuclear EOS protein into the endogenous fgf10b locus. We found fgf10b:nEOS to be robustly expressed in the forming gills, with expression becoming

167 progressively restricted to the tips of gill filaments over time, similar to endogenous fgf10b expression (Fig. S10, S14). We then used UV light to convert fgf10b:nEOS fluorescence from green to red in a small number of filaments at $7 \mathrm{dpf}$ and observed contribution to gill chondrocytes and pillar cells 3 days later, with new fgf10b:nEOS cells (i.e. green only) being generated at the tips of growing filaments (Fig. 2i). Similar results were seen in adult gill filaments (Fig. S14). These data support fgf10b+ cells being progenitors for gill-specific cell types from larval through adult 173 stages.

To understand how CNCC mesenchyme changes from embryogenesis to adulthood, we next interrogated patterns of gene usage and chromatin accessibility (Fig. 2j, Fig. S15-16, Table S2). Gene ontogeny (GO) analysis of ectomesenchyme at 1.5 and $2 \mathrm{dpf}$ revealed terms linked to cell division and metabolism, consistent with early expansion of this population. We also find enrichment of transcription factors for early ectomesenchyme (dlx2a, twist1a, nr2f6b) and arch patterning (pou3f3b, hand2), as well as transcription factor binding motifs for several types of nuclear receptors, in accordance with known roles of Nr2f members in ectomesenchyme development ${ }^{19}$. The hyal4+ population contains skeletal-associated terms (collagen fibril 183 organization, skeletal system development, regulation of ossification, cartilage development), 184 consistent with being a common progenitor for cartilage, tendon, ligament, and bone in 185 pseudotime analysis. The hyal4+ population is enriched for transcription factors implicated in 186 perichondrium biology (mafa, foxp2, foxp4) $)^{23,24}$ and cartilage formation (barx1, sox6, emx2) ${ }^{25-27}$, 187 and motifs for Bmp signaling (SMAD) and transcription factors (NFAT, RUNX) known to regulate 188 cartilage and bone ${ }^{28}$. For gill fgf10a/b+ progenitors, we recover terms for general growth (e.g. 
translation, cellular biosynthetic process), response to Fgf signaling, and respiratory system development, consistent with lineage tracing showing fgf10b:nEOS-labeled cells giving rise to gill respiratory cell types through adult stages. We also observe enrichment of gata2a, gata3, and GATA motif accessibility, suggesting important roles of Gata factors in gill-specific lineages.

In contrast to hyal4+ and $f g f 10 a / b+$ populations that display hallmarks of progenitors, the $c x c / 12 a+$ stromal population is associated with terms for regeneration, response to injury and wounding, negative regulation of the Wnt signaling pathway, and, particularly at adult stages, response to stress and modulation of the immune response. This population is enriched for osr1, early response genes of the Fos/Jun family, C/EBP family members implicated in response to inflammation ${ }^{29}$, and egr1 that has recently been linked to injury-induced regenerative responses across the animal kingdom ${ }^{30}$. Recovery of motifs for STAT and C/EBP also point to immune system interactions. As $\mathrm{Cxcl12+}$ stromal cells in murine bone marrow have been shown to only contribute to osteoblasts during bone regeneration ${ }^{31}$, it will be interesting to test whether the $c x c / 12 a+$ stromal population in animals such as zebrafish that lack bone marrow also plays a role in skeletal regeneration ${ }^{32}$.

\section{Highly resolved embryonic spatial expression domains from integrated datasets}

207 We next sought to understand the developmental origins of distinct cell types and lineage programs in CNCC ectomesenchyme. To do so, we first examined the ability of integrated transcriptomic and chromatin accessibility datasets to predict the expression patterns of potential ectomesenchyme patterning genes at $1.5 \mathrm{dpf}$, a stage before overt cell type differentiation.

211 Compared to scRNAseq (Fig. 3a) or snATACseq alone (Fig. S17), SnapATAC pseudo-multiome

212 analysis (Fig. 3b) was better able to separate CNCCs along the major positional axes, including 213 the dorsal-ventral axis and the anterior-posterior axis (frontonasal, mandibular (arch 1), hyoid 214 (arch 2), branchial (arch 3-6), and tooth-bearing (arch 7)). 
Comparison of the predicted SnapATAC expression of known region-specific genes - pou3f3b

217 (dorsal arches 1 and 2), dlx5a (intermediate arches), hand2 (ventral arches), meis $2 b$ (arch 7),

and pitx1 (oral mandibular) ${ }^{25,33,34}$ - revealed tight correlation to reported expression, including

zebrafish-specific overlap of $d l \times 5 a$ and hand2 in the mandibular arch (Fig. 3d). We also identified

a previously unappreciated oral-aboral axis of the mandibular arch in zebrafish, marked by pitx1

and $n r 5 a 2$ respectively, which we validated by in situ hybridization for $n r 5 a 2$ (Fig. 3e). Re-

examination of genes identified from previous bulk RNA sequencing of zebrafish arches further

revealed strong correlation of SnapATAC domains with reported expression for 23 of 27 genes

(Fig. S18), with SnapATAC suggesting frontonasal and tooth-domain expression for two genes

previously annotated as false positives ${ }^{25}$. We also observed correlation of the transcription factor

binding motifs enriched in cluster-specific accessible chromatin with the activities of transcription pharyngeal arch stages.

\section{Chromatin accessibility predicts cell type competency in early arches}

233 We next sought to understand how the establishment of cell fate competency is linked to the earlier activity of arch patterning genes. To do so, we first computed unique patterns of chromatin accessibility ("peaks") for each cell cluster at $14 \mathrm{dpf}$ (Fig. 4a, Table S3). Modules of the top enriched peaks for each cell type were then mapped onto UMAP projections of SnapATAC data at 1.5, 2, 3, and $5 \mathrm{dpf}$ (Fig. S19). To understand when cluster-specific peaks become established, as well as cluster relatedness, we developed the bioinformatics pipeline "Constellations". First, 
241 chromatin accessibility (a proxy for cell type competency). We then computed the relatedness of

242 peak module projections in two dimensions for each mapped cluster at each stage (Fig. 4b).

243 Analysis of cell competency trajectories shows that cell types can be grouped into five main

244 classes: skeletogenic cells (including hyal4+ perichondral and postnb+ periosteal cells), stromal

245 cells, dermal fibroblasts, gill cell types, and cartilage. Constellations analysis also reveals a

246 temporal order of cell type competency establishment, with unique chromatin accessibility for

247 cartilage and dermal fibroblast lineages emerging at $1.5 \mathrm{dpf}$; bone and perichondrium at $2 \mathrm{dpf}$;

248 and periosteum, tendon and ligament, and gill progenitors and pillar cells at $3 \mathrm{dpf}$ (Fig. 4c). This

249 analysis suggests that chromatin accessibility prefiguring diverse CNCC cell types is

progressively established rather than being inherited from earlier multipotent CNCCs.

\section{Constellations analysis reveals candidate transcription factors for lineage priming}

To discover potential transcription factors for establishing cell type competency, we analyzed the

Constellations dataset for transcription factors whose expression and predicted binding motifs

were co-enriched in particular clusters. We identified 287 transcription factor expression/motif were highly enriched in the cartilage trajectory, and LEF1/lef1 in the dermal fibroblast trajectory

(Fig. 5a). Projection of FOX motifs and merged Fox gene activity (foxc1a, foxc1b, foxf1, foxf2a,

259 foxf2b) and LEF1/lef1 onto SnapATAC UMAPs at $1.5 \mathrm{dpf}$ reveals close correlation to mapping of

260 the $14 \mathrm{dpf}$ peak modules for cartilage and dermal fibroblasts at this stage (Fig. 5b,c), as well as

261 the known fate map of cartilage precursors in the $\operatorname{arches}^{35}$ (Fig. 5d,e). This confirms genetic evidence for roles of Foxc1 and Foxf1/2 in cartilage formation in zebrafish and mouse ${ }^{36,37}$, and 
We also find GATA3/gata3 to be highly enriched in gill populations, with SnapATAC UMAP projections of GATA3 motif and gata3 gene body activity at 5 dpf correlating with 14 dpf gill progenitor peaks (Fig. 5f). The enrichment of ETS2/ets2, which plays a role in endothelial

271 development ${ }^{38}$, in the gill pillar trajectory is consistent with ETS factors driving a mesenchyme-to-

272 endothelia transition during formation of these vascular cells. Skeletogenic trajectories are

273 uniquely marked by IRF8/irf8. Whereas loss of bone in mouse $/$ Iff ${ }^{-/-}$mutants has been attributed

274 to increased osteoclastogenesis ${ }^{39}$, our analysis suggests that Irf8 may also have an early function

275 in priming the skeletal lineage. Enrichment of CEBPA/cebpa in stromal trajectories may reflect the immunomodulatory role of this mesenchymal population ${ }^{29}$. These findings show the power of Constellations analysis to reveal potential factors for establishing regional chromatin accessibility important for later cell type differentiation.

\section{Gill-specific lineages distinguished by early Gata3 activity}

281 Given the selective enrichment of GATA3 motifs and gata3 activity in gill lineages, we further 282 investigated the presence of a Gata3 regulatory circuit directing CNCCs to gill fates. Whereas 283 previous work had shown that gata3 is expressed in and required for initial gill bud formation in 284 zebrafish, larval lethality had precluded analysis of gill subtype differentiation ${ }^{40}$. We find gata3 expression to be maintained in gill populations through adult stages in scRNAseq data, which we validated by in situ hybridization at $14 \mathrm{dpf}$ and 2 years of age (Fig. S21). We then identified a noncoding region $\sim 143 \mathrm{~kb}$ downstream of the gata3 gene, itself containing a predicted GATA3 binding site, that was selectively accessible in posterior arch CNCCs by $3 \mathrm{dpf}$, gill progenitors and pillar cells by $5 \mathrm{dpf}$, and gill cartilage cells by $14 \mathrm{dpf}$ (Fig. 6a, Fig. S22). This gata3-P1 element was sufficient to drive highly restricted GFP expression in posterior arch CNCCs starting at $1.5 \mathrm{dpf}$, 
294 Gill cartilage has a markedly distinct expression and chromatin accessibility profile from hyaline

295 cartilage of the jaw, as shown by selective expression of ucmaa in gill cartilage versus ucmab in

296 hyaline cartilage (Fig. S23). We identified a non-coding region $\sim 5 \mathrm{~kb}$ upstream of the ucmaa gene

297 that was selectively accessible in gill cartilage starting at $14 \mathrm{dpf}$ and contained a predicted GATA3-

298 binding site (Fig. 6b, Fig. S22). This ucmaa-P1 element drives highly restricted GFP expression

299 in gill chondrocytes at 11 and $23 \mathrm{dpf}$, in contrast to a previously described $u c m a b$ enhancer ${ }^{28}$

300 driving GFP expression in hyaline but not gill cartilage (Fig. 6f, Fig. S23). Although functional assays are needed to confirm Gata3 dependence, our findings are consistent with GATA factors establishing a positive autoregulatory circuit in posterior arch CNCCs that maintains gata3 expression and promotes the later differentiation of gill-specific cell types (Fig. $6 \mathrm{~g}$ ).

\section{Conclusion}

306 Integration of transcriptome and chromatin accessibility data of the CNCC lineage has allowed us

307 to connect patterning along major development axes to the emergence of the wide diversity of 308 CNCC-derived cell types. Rather than lineage-specific chromatin accessibility being an intrinsic 309 property of CNCCs, our Constellations analysis points to the progressive remodeling of chromatin underlying diverse cell type differentiation. Roles for inductive signaling in establishing enhancer

311 accessibility in post-migratory arch CNCCs would help explain reports of mesodermal cells contributing to classically considered CNCC structures such as the skate gill skeleton ${ }^{7}$. Further, retrograde mapping of cell type-specific chromatin accessibility, combined with our highly resolved atlas of pharyngeal arch gene expression, reveals candidate transcription factors priming distinct CNCC lineages. Consistent with recent reports of organ-specific fibroblast heterogeneity ${ }^{41}$, we also uncover a CNCC-derived dermal fibroblast population characterized by

317 Phe/Tyr metabolism genes, which may be induced by early Wnt/Lef1 activity. Expression of some 318 of the same Phe/Tyr catabolic genes is observed in a subset of axolotl limb fibroblasts ${ }^{42}$, with the 
blackening of bone and cartilage in Alkaptonuria patients defective in Phe/Tyr breakdown suggesting general roles for these specialized dermal fibroblasts in protecting the skeleton. In the

321 gill region, we identify a fgf10-expressing progenitor population characterized by sustained Gata3

322 activity, with later emergence of Ets2 activity in pillar cells providing a potential mechanism for the

323 mesenchyme-to-endothelia transition of these specialized vascular cells. The presence of a 324 similar Fgf10-expressing mesenchyme population in the mammalian lung ${ }^{43}$ raises the possibility 325 that an ancestral CNCC-derived gill respiratory program may have been co-opted by the mesoderm during later lung evolution. Single-cell profiling of transcriptome and chromatin accessibility across time thus provides a blueprint for understanding the diversification and postembryonic production of the huge variety of CNCC-derived cell types throughout the head.

\section{Materials and methods}

Zebrafish lines

332 The Institutional Animal Care and Use Committee of the University of Southern California approved all animal experiments (Protocol 20771). Published lines include Tg(Mmu.Sox10-

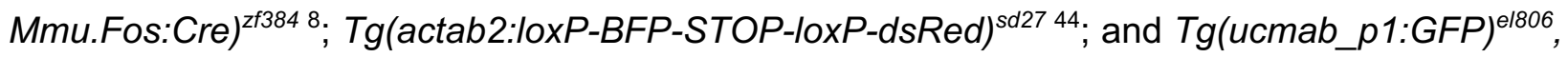
$\operatorname{Tg}$ (fli1a:eGFP) $^{y 1}$, and $\operatorname{Tg}\left(\right.$ sox10:DsRedExpress) ${ }^{e / 1028}$. Five transgenic lines were generated as part of this study: Tg(fgf10b:nEOS) $)^{e / 865}, \quad \operatorname{Tg}(\text { gata3_p1:GFP })^{e / 857}, \quad \operatorname{Tg}\left(g a t a 3 \_p 1: G F P\right)^{e, 858}$,

337 Tg(ucmaa_p1:GFP) $)^{e / 851}$ and Tg(ucmaa_p1:GFP) ${ }^{\text {el854}}$. The fgf10b:nEOS knock-in line was made 338 using CRISPR/Cas9-based integration ${ }^{45}$. Three gRNAs targeting sequences upstream of the 339 fgf10b translational start site (5'-CATGATAACCCTTCCTAGAT-3', 5'340 GAGCTCTTTGATAGCGGGCT-3', and 5'-GTTGAGCAGCATGTCCCATG-‘3) were co-injected at $341100 \mathrm{ng} / \mathrm{uL}$ into wild-type embryos with Cas9 RNA (100 ng/uL), an mbait-NLS-EOS plasmid (20 $342 \mathrm{ng} / \mathrm{uL})^{46}$, and the published gRNA targeting the mbait sequence ${ }^{45}$ to linearize the plasmid. A 343 germline founder was identified based on nEOS fluorescence in the progeny of injected animals. 344 For enhancer transgenic lines, we synthesized peaks for gata3 (chr4:24918100-24918770) and 
ucmaa (chr4:7836670-783720) using iDT gBlocks and cloned these into a modified pDest2AB2 construct containing E1b minimal promoter, GFP, and an eye-CFP selectable marker ${ }^{28}$ using In-

347 Fusion cloning (Takara Bio). We injected plasmids and Tol2 transposase RNA (30 ng/uL each)

348 into one-cell stage zebrafish embryos, raised these animals, and screened for founders based on

349 eye CFP expression in the progeny. Two independent germline founders were identified for each 350 that showed similarly specific activity in the gills.

\section{In situ hybridization and immunohistochemistry}

All samples were prepared by fixation in $4 \%$ paraformaldehyde and embedded in paraffin, with decalcification for one week in $20 \%$ EDTA if over $14 \mathrm{dpf}$. All in situ patterns were confirmed in at least 3 independent animals. RNAscope probes were synthesized by Advanced Cell Diagnostics in channels 1 through 4. Channel 1 probes: ifitm5, ucmaa, col10a1a. Channel 2 probes: postnb, myh11a, cxcl12a, sp7, gata3. Channel 3 probes: pah, lum, fgf10b, sox9a. Channel 4 probe: hyal4, acta2, ncam3. Paraformaldehyde-fixed paraffin-embedded sections were deparaffinized, and the RNAscope Fluorescent Multiplex V2 Assay was performed according to manufacturer's protocols with the ACD HybEZ Hybridization oven. Colorimetric in situ hybridization was performed as described ${ }^{32}$. The $h p d b$ riboprobe was generated by cloning a purchased gBlock fragment designed from transcript hpdb-201 using nucleotides 679-1395 (tggatga...gactccc) into pCRBluntll-TOPO (Life Technologies). The $n r 5 a 2$ riboprobe was generated by PCR amplification of cDNA with primers 5'-ATGGGGAACAGGGGCATATG-3' and 5'-AGGGGTCGGGATACTCTGAT-3', the ucmaa riboprobe with primers 5'-TGGTACCAGCTCAAGACACT-3' and 5'ATAGTACTGGCGGTGGTGAG-3', the ucmab riboprobe with primers 5'ATGTCCTGGACTCAACCTGC-3' and 5'-GTTATCTCCCAGCGTGTCCA-3', and the 
Dissected heads from converted Sox10:Cre; actab2:IoxP-BFP-STOP-loxP-dsRed fish were

pCR-BluntII-TOPO plasmids were linearized by restriction digest (enzyme dependent on direction of blunt insertion), and RNA probe was synthesized using either T7 or Sp6 polymerase (Roche) depending on direction of blunt insertion. Immunohistochemistry for dsRed was performed with a 7 minute $-20^{\circ} \mathrm{C} 100 \%$ acetone target retrieval and blocking in $2 \%$ normal goat serum (Jackson ImmunoResearch, cat. no. 005-000-121). Primary antibodies include rabbit anti-mCherry (1:100, Rockland Immunochemicals, cat. no. RL600-401-P16) and rabbit anti-mCherry (1:100, Novus Biologicals, cat. no. NBP2-25157) used at the same time. The secondary antibody was goat antirabbit Alexa Fluor 546.

\section{Imaging}

Confocal images of whole-mount or section fluorescent in situ hybridizations and live images of transgenic fish were captured on a Zeiss LSM800 microscope using ZEN software. Colorimetric in situs were imaged on a Zeiss AxioScan Z.1 For fgf10:nEOS experiments, we used the ROI function on the confocal microscope to specifically convert nEOS-expressing cells in the gill filaments of live animals using targeted UV irradiation, prior to the emergence of gill filament cartilage. At the specified days post-conversion, we euthanized the animal, fixed it in $4 \%$ PFA for 1 hour, and dissected the gill arches. We stained the gills with DRAQ5 nuclear dye (Abcam) for 30 min and imaged at $40 \mathrm{X}$ to locate converted cells. For all transgenic imaging experiments, expression patterns were confirmed in at least 5 independent animals.

\section{Single-cell analysis and statistics}

scRNAseq and snATACseq library preparation and alignment incubated in fresh Ringer's solution 5-10 min, followed by mechanical and enzymatic dissociation by pipetting every 5 minutes in protease solution (0.25\% trypsin (Life Technologies, 15090-046), 
$1 \mathrm{mM}$ EDTA, and $400 \mathrm{mg} / \mathrm{ml}$ Collagenase D (Sigma, 11088882001) in PBS) and incubated at $\mathrm{mM} \mathrm{CaCl} 2$ and $30 \%$ fetal bovine serum (FBS) in PBS). Cells were pelleted (2000 rpm, 5 min, 4 ${ }^{\circ} \mathrm{C}$ ) and resuspended in suspension media (1\% FBS, $0.8 \mathrm{mM} \mathrm{CaCl} 2,50 \mathrm{U} / \mathrm{ml}$ penicillin, and 0.05 $\mathrm{mg} / \mathrm{ml}$ streptomycin (Sigma-Aldrich, St. Louis, MO) in phenol red-free Leibovitz's L15 medium (Life Technologies)) twice. Final volumes of $500 \mu \mathrm{l}$ resuspended cells were placed on ice and sorted by fluorescence activated cell sorting (FACS) to isolate live cells that excluded the cytoplasmic stain Zombie green (BioLegend, 423111). For scRNAseq library construction, barcoded single-cell cDNA libraries were synthesized using 10X Genomics Chromium Single Cell 3' Library and Gel Bead Kit v.2 per manufacturer's instructions. Libraries were sequenced on Illumina NextSeq or HiSeq machine at a depth of at least 1,000,000 reads per cell for each library. Read2 was extended to 126 cycles for higher coverage. Cellranger v3.0.0 (10X Genomics) was used for alignment against GRCz11 (built with GRCz11.fa and GRCz11.98.gtf) and gene-by-cell count matrix generation with default parameters.

For snATACseq library construction, we used the same cell dissociation and sorting protocol as for scRNAseq, with isolation of live cells that excluded the cytoplasmic stain Zombie green (BioLegend, 423111) and collected live cells in 0.04\% BSA/PBS. Nuclei isolation was performed per manufacturer's instructions (10X Genomic, protocol CG000169). Cells were incubated with Iysis buffer on ice for $90 \mathrm{~s}$, followed by integrity check of nuclei under fluorescence microscope with DAPI before library synthesis. Barcoded single-nuclei ATAC libraries were synthesized using 415 10X Genomics Chromium Single Cell ATAC Reagent Kit v1.1 per manufacturer's instructions. 416 Libraries were sequenced on Illumina NextSeq or HiSeq machine at a depth of at least 75,000 417 reads per nucleus for each library. Both read1 and read2 were extended to 65 cycles. Cellranger 418 ATAC v1.2.0 (10X Genomics) was used for alignment against genome (built with GRCz11.fa, 419 JASPAR2020, and GRCz11.98.gtf), peak calling, and peak-by-cell count matrix generation with 420 default parameters.

421 We included biological replicates at several stages to test the reproducibility of library preparation 422 and increase depth of data. For scRNAseq, we performed two replicates at 5 and $14 \mathrm{dpf}$, and 
423 three replicates at 3 and $150 \mathrm{dpf}$. For snATACseq, we performed two replicates at 2, 3, and 14

$424 \mathrm{dpf}$.

To refine the peak profile for better representation of diverse cell types across libraries, we performed a second round of peak calling using package Snaptools (v1.2.7) and SnapATAC (v1.0.0) ${ }^{15}$. We first removed low-quality cell and cell doublets by setting cutoffs based on percentage of reads in peaks ( $>30$ for $60 \mathrm{dpf},>45$ for $210 \mathrm{dpf}$, and $>50$ for the rest) and fragment number within peaks $(5,000-30,000$ for $5 \mathrm{dpf}, 1,000-11,000$ for $14 \mathrm{dpf}$, and 1,000 - 20,000 for the rest). Potential cell debris or low-quality cells were removed by setting hard fragments-in-peak number cutoffs. Using the SnapATAC package, we then generated "pseudo-multiome" data at each stage. To recover every aligned fragment, we binned the genome into $5 \mathrm{~kb}$ sections and constructed the bin-by-cell matrices (bmats) for each library by Snaptools from the positionalsorted bam files generated by Cellranger ATAC v1.2.0. The cells were filtered, dimensionally reduced by diffusion map, and clustered with inputs of the first 34 dimensions followed the SnapATAC vignette. The specific peaks were called for each cluster by the wrapped MACS2 function in SnapATAC with parameter gsize $=1.5 e 9$, shift $=100$, ext $=200$, and qval $=5 e-2$. The

440 finalized and refined peak profile was derived by collapsing and merging all 175 individual peak 441 files to 445,307 peaks. To impute the gene activity with the corresponding scRNAseq data, the bmats of each time point were used to calculate gene-activity-by-cell matrices (gmats) by

443 SnapATAC. The gmats were used to find anchors within the scRNAseq data at the same time 444 point by Seurat. We then transferred the expression data from scRNAseq through the anchors to 445 derive the imputed gene-activity-by-cell matrices for each time point.

447 The count matrices of both scRNAseq and snATACseq data were analyzed by R package Seurat 448 (v3.2.3) and Signac (v1.0.0). The count matrices of each sample were aggregated where 449 replicates were available. For scRNAseq data, the matrices are normalized (NormalizeData) and 
scaled for the top 2,000 variable genes (FindVariableFeatures and ScaleData). The scaled matrices were dimensionally reduced to 50 principal components (60 components for $150 \mathrm{dpf}$ ), and then subjected to neighbor finding (FindNeighbors, $k=20$ ) and clustering (FindClusters, resolution $=0.8$ ). The data were visualized through UMAP with 50 principal components as input. For snATACseq data, the matrices are dimensionally reduced to 30 latent semantic indices (LSIs) through RunTFIDF and RunSVD functions. The neighbor finding, clustering, and visualization are performed as for scRNAseq data (algorithm $=3$ for FindClusters) with input of the second to thirtieth LSIs. To calculate the motif accessibility, the enrichment of motifs in JASPAR2020 ${ }^{47}$ was calculated by chromVAR ${ }^{48}$ through function RunChromVar. To test the enriched genes and gene activities in both scRNAseq and snATACseq data as shown in Table S1, two-sided likelihoodratio test is performed through FindAllMarkers function (min.pct $=0.25$ ) with cutoff of adjusted $p$ value smaller than 0.001

\section{STITCH network construction and force directed layout}

To identify the overall cell trajectories in our scRNAseq and snATACseq data, we used the STITCH algorithm ${ }^{17}$ to construct cell neighbor networks. As the dimensional reduction space of snATACseq data are LSIs, we modified the stitch_get_knn and stitch_get_link function of the STITCH package to make it compatible to LSI. For stitch_get_knn function of snATACseq data, we used the LSI matrix to find the $\mathrm{k}$ nearest neighbor of each cell for each time point. For stitch_get_link function of snATACseq data, we projected the LSI space of time point $t$ to time point $t-1$ by solving the right orthogonal matrix of the singular vector decomposition (SVD) for $t-1$. SVD $\left(M=U \Sigma V^{T}\right)$ is the initial step of latent semantic analysis where $M$ is the peak-by-cell matrix and $U$ will be later transformed to LSI. For $t$ and $t-1$, we have $M_{t}=U_{t} \Sigma_{t} V^{T}{ }_{t}$ and $M_{t-1}=U_{t-1} \Sigma_{t-1} V_{t-1}^{T}$. To project the space from $t$ to $t-1$, we derived a projected $U_{t-1}$ as $U_{t-1}^{p}$ through solving the equation $M_{t-1}=U_{t-1}^{p} \Sigma_{t} V_{t}^{T}$. Both $U_{t}$ and $U_{t-1}^{p}$ were further combined, normalized, and subjected to the default neighbor finding as stitch_get_link. To visualize the STITCH networks of both scRNAseq and snATACseq data, we used the force directed layout by ForceAtlas2 in Gephi (v0.9.2) to derive the 2-dimensional layouts. 


\section{Pseudotime analysis}

480

481

482

483

484

485

486

487

488

489

490

491

492

493

494

495

496

497

498

499

500

501

502

We used the R package monocle3 (v0.2.3.0) to predict the pseudotemporal relationships within skeletogenic or gill populations. We first merged 5 and $14 \mathrm{dpf}$ scRNAseq data, including an additional scRNAseq library of sox10>dsRed+ cells sorted from the dissected ceratohyal endochondral bone at $14 \mathrm{dpf}$ (to further enrich skeletogenic populations), and performed clustering and dimensionality reduction. After removing dermal fibroblast $(p a h+)$ and teeth (spock3+) populations, we placed hoxb3a+/gata3+ cells into a "gill" cluster and all other cells into a "jaws" cluster. Cell paths were predicted by the learn_graph function of monocle3. We set the origin of the cell paths based on the enriched distribution of $5 \mathrm{dpf}$ cells.

Gene ontology, motif family, and TF analysis of CNCC mesenchyme

Analysis was performed on ectomesenchyme, perichondrium, gill progenitor, and stromal populations at each stage based on markers from the scRNAseq data (Fig. S1-7). The enriched genes of each cluster are tested by running a two-sided Wilcoxon rank sum test against all other clusters using an adjusted $p$ value $\leq 0.001$. These enriched gene sets are subjected to gene ontology analysis for terms of biological processes (BP) by R package ViSEAGO (v1.2.0) ${ }^{49}$. The heatmap is generated by GOterms_heatmap function using values of log10(adjusted p.value). To generate the heatmap of motif families for each cluster, we first averaged and aggregated the motif accessibilities for each cell according to the motif family by TRANSFAC ${ }^{50}$. The means of each motif family are used for the heatmap. To generate the heatmap of TFs for each cluster, we subsetted the TFs from the enriched gene sets, and used the mean of each TF for every cluster for the heatmap.

\section{Constellations analysis and calculation of cluster skewedness and correlation}


503 The tissue module scores of the snATACseq data were calculated based on the enriched peak

504 sets and their module scores for each cluster identified at $14 \mathrm{dpf}$ by $\mathrm{R}$ packages Seurat and

505 Signac. The enriched peak sets were calculated by the FindAllMarkers function using two-sided

506 likelihood ratio test with fragment numbers in peak region as latent variables. We used the peaks

507 with adjusted $p$ values smaller than 0.001 as the enriched peaks for a cluster. As there are 23

508 clusters (tissues) identified at $14 \mathrm{dpf}$, we ended up with 23 peak sets, which we applied to

509 calculate the tissue module scores to earlier time points $(1.5,2,3$, and 5 dpf) using

510 AddChromatinModule function. To determine whether a tissue score at a time point distributes in

511 a statistically significant, and hence biologically interesting, way, we calculated the skewedness

512 of the distribution of a tissue score by the R package parameter (v0.12.0). We considered a tissue

513 score to be distributed in a meaningful way if it was strongly right skewed by a hard cutoff of

514 skewedness greater than 1. For $1.5 \mathrm{dpf}$, the cutoff of skewedness was lowered to 0.4 to

515 accommodate overall lower skewedness at that time point, but with additional filter of max module

516 score $>15$ to avoid tissue module scores with extremely low values.

517 To profile the relationship of all tissue scores, we constructed a distance matrix of all 23 tissue

518 module scores across all the time points $(1.5,2,3,5$, and $14 \mathrm{dpf})$. For the distance $D$ between

519 score of tissue $A$ at time point $t_{1}$ and score of tissue $B$ at time point $t_{2}$, the distance $D$ can be

520 described as $D=D$ (tissue) $+($ a $\times D$ (time point $))$. $D$ (tissue) stands for the distance between the

521 score of tissue $A$ and $B$ by averaging the Euclidian distance between score $A$ and $B$ at time point

$522 t_{1}$ and the Euclidian distance between score $A$ and $B$ at time point $t_{2}$. $D$ (time point) stands for the

523 distance between time point $t_{1}$ and $t_{2}$ derived by the distance between the dendrogram of all the

524 tissue scores at $t_{1}$ and the dendrogram at $t_{2}$. Since $D$ (time point) is relatively smaller than

$525 D$ (tissue), we multiply $D$ (time point) by 8 to make the distance between time points comparable

526 to the distance between tissue scores. The distance matrix was dimensionally reduced and

527 visualized by UMAP.

528 To detect the potential factors that contribute to the patterning of tissue-specific peaks, we 529 performed linear regression of each tissue module score against all motif accessibilities and the 530 gene activities of transcription factors. We used ZFIN and JASPAR2020, converted by homology 
531 data from $\mathrm{MGI}$, to build up a list of transcription factors in zebrafish. We then curated and paired

532 every motif in JASPAR2020 with its potential binding transcription factors. The coefficients of 533 regression results were used as indications of whether a motif or transcription factor is positively

534 correlated with a tissue module score with upper cutoff of adjusted $p$ value 0.05 . We transformed 535 the coefficients of all the negative related motifs and transcription factors to 0 to filter out irrelevant 536 motifs and transcription factors. To visualize the correlation of each pair of motif and transcription 537 factor, we plotted the coefficient magnitudes of motifs by dot sizes and transcription factor gene 538 body activities by a red color scale on Constellation maps.

\section{Acknowledgments}

541 We thank Megan Matsutani and Jennifer DeKoeyer Crump for fish care, Jeffrey Boyd and the 542 BCC FACS core, the CHLA Sequencing core, the USC HPC computing core, and Andrew 543 McMahon, Yang Chai, and Unmesh Jadhav for helpful comments on the manuscript.

\section{Competing interests}

$546 \quad$ No competing interest declared.

\section{Funding}

549 Funding was provided by the National Institute of Health (NIDCR R35 DE027550 to J.G.C.; 550 NIDCR K99 DE029858 to P.F; NIDCR F31 DE029682-02 to C.A.; NICHD T32 HD060549 to M.T.).

\section{Data availability}

553 The data that support the findings of this study are available from the corresponding author upon 554 reasonable request. Processed and raw sequencing data have been deposited at GEO as 555 GSE178969. 


\section{Author Contributions}

557 P.F., K.-C.T., M.T., C.A., H.-J.C., J.S., N.N., and J.G.C. performed the experiments. J.G.C.

558 oversaw the project and wrote the manuscript.

\section{References}

561

562

563

564

565

566

567

568

569

570

571

572

573

574

575

576

577

578

579

580

581

582

583

584

585

586

587

588

589

590

591

592

593

594

595

596

597

598

599

600

601

1 Platt, J. B. Ectodermic origin of the cartilages of the head. Anat. Anz. 8, 506-509 (1893).

2 Mongera, A. et al. Genetic lineage labeling in zebrafish uncovers novel neural crest contributions to the head, including gill pillar cells. Development 140, 916-925, doi:10.1242/dev.091066 (2013).

3 Dupin, E., Calloni, G. W., Coelho-Aguiar, J. M. \& Le Douarin, N. M. The issue of the multipotency of the neural crest cells. Dev Biol 444 Suppl 1, S47-S59, doi:10.1016/j.ydbio.2018.03.024 (2018).

4 Baroffio, A., Dupin, E. \& Le Douarin, N. M. Common precursors for neural and mesectodermal derivatives in the cephalic neural crest. Development 112, 301-305 (1991).

5 McGonnell, I. M. \& Graham, A. Trunk neural crest has skeletogenic potential. Curr Biol 12, 767-771, doi:10.1016/s0960-9822(02)00818-7 (2002).

6 Simoes-Costa, M. \& Bronner, M. E. Reprogramming of avian neural crest axial identity and cell fate. Science 352, 1570-1573, doi:10.1126/science.aaf2729 (2016).

7 Sleight, V. A. \& Gillis, J. A. Embryonic origin and serial homology of gill arches and paired fins in the skate, Leucoraja erinacea. Elife 9, doi:10.7554/eLife.60635 (2020).

8 Kague, E. et al. Skeletogenic fate of zebrafish cranial and trunk neural crest. PLoS One 7, e47394, doi:10.1371/journal.pone.0047394 (2012).

9 Mitchell, J. M. et al. The alx3 gene shapes the zebrafish neurocranium by regulating frontonasal neural crest cell differentiation timing. Development, doi:10.1242/dev.197483 (2021).

10 Williams, R. M. et al. Reconstruction of the Global Neural Crest Gene Regulatory Network In Vivo. Dev Cell 51, 255-276 e257, doi:10.1016/j.devcel.2019.10.003 (2019).

11 Soldatov, R. et al. Spatiotemporal structure of cell fate decisions in murine neural crest. Science 364, doi:10.1126/science.aas9536 (2019).

$12 \mathrm{Xu}, \mathrm{J}$. et al. Hedgehog signaling patterns the oral-aboral axis of the mandibular arch. Elife 8, doi:10.7554/eLife.40315 (2019).

13 Yuan, Y. et al. Spatiotemporal cellular movement and fate decisions during first pharyngeal arch morphogenesis. Sci Adv 6, doi:10.1126/sciadv.abb0119 (2020).

14 Zalc, A. et al. Reactivation of the pluripotency program precedes formation of the cranial neural crest. Science 371, doi:10.1126/science.abb4776 (2021).

15 Fang, R. et al. Comprehensive analysis of single cell ATAC-seq data with SnapATAC. Nat Commun 12, 1337, doi:10.1038/s41467-021-21583-9 (2021).

16 Fernandez-Canon, J. M. et al. The molecular basis of alkaptonuria. Nat Genet 14, 19-24, doi:10.1038/ng0996-19 (1996).

17 Wagner, D. E. et al. Single-cell mapping of gene expression landscapes and lineage in the zebrafish embryo. Science 360, 981-987, doi:10.1126/science.aar4362 (2018).

18 Tang, W., Martik, M. L., Li, Y. \& Bronner, M. E. Cardiac neural crest contributes to cardiomyocytes in amniotes and heart regeneration in zebrafish. Elife 8, doi:10.7554/eLife.47929 (2019).

19 Barske, L. et al. Essential Role of Nr2f Nuclear Receptors in Patterning the Vertebrate Upper Jaw. Dev Cell 44, 337-347 e335, doi:10.1016/j.devcel.2017.12.022 (2018). 
602

603

604

605

606

607

608

609

610

611

612

613

614

615

616

617

618

619

620

621

622

623

624

625

626

627

628

629

630

631

632

633

634

635

636

637

638

639

640

641

642

643

644

645

646

647

648

649

650

651

652

653

20 Cao, J. et al. The single-cell transcriptional landscape of mammalian organogenesis. Nature 566, 496-502, doi:10.1038/s41586-019-0969-x (2019).

21 Bandyopadhyay, A., Kubilus, J. K., Crochiere, M. L., Linsenmayer, T. F. \& Tabin, C. J. Identification of unique molecular subdomains in the perichondrium and periosteum and their role in regulating gene expression in the underlying chondrocytes. Dev Biol 321, 162-174, doi:10.1016/j.ydbio.2008.06.012 (2008).

22 Colnot, C., Lu, C., Hu, D. \& Helms, J. A. Distinguishing the contributions of the perichondrium, cartilage, and vascular endothelium to skeletal development. Dev Biol 269, 55-69, doi:10.1016/j.ydbio.2004.01.011 (2004).

23 Huang, W., Lu, N., Eberspaecher, H. \& De Crombrugghe, B. A new long form of c-Maf cooperates with Sox9 to activate the type II collagen gene. J Biol Chem 277, 50668-50675, doi:10.1074/jbc.M206544200 (2002).

24 Zhao, H. et al. Foxp1/2/4 regulate endochondral ossification as a suppresser complex. Dev Biol 398, 242-254, doi:10.1016/j.ydbio.2014.12.007 (2015).

25 Askary, A. et al. Genome-wide analysis of facial skeletal regionalization in zebrafish. Development 144, 2994-3005, doi:10.1242/dev.151712 (2017).

26 Lefebvre, V., Li, P. \& de Crombrugghe, B. A new long form of Sox5 (L-Sox5), Sox6 and Sox9 are coexpressed in chondrogenesis and cooperatively activate the type II collagen gene. EMBO J 17, 5718-5733, doi:10.1093/emboj/17.19.5718 (1998).

27 Nichols, J. T., Pan, L., Moens, C. B. \& Kimmel, C. B. barx1 represses joints and promotes cartilage in the craniofacial skeleton. Development 140, 2765-2775, doi:10.1242/dev.090639 (2013).

$28 \mathrm{Xu}, \mathrm{P}$. et al. Foxc1 establishes enhancer accessibility for craniofacial cartilage differentiation. Elife 10, doi:10.7554/eLife.63595 (2021).

29 Poli, V. The role of C/EBP isoforms in the control of inflammatory and native immunity functions. J Biol Chem 273, 29279-29282, doi:10.1074/jbc.273.45.29279 (1998).

30 Gehrke, A. R. et al. Acoel genome reveals the regulatory landscape of whole-body regeneration. Science 363, doi:10.1126/science.aau6173 (2019).

31 Matsushita, Y. et al. A Wnt-mediated transformation of the bone marrow stromal cell identity orchestrates skeletal regeneration. Nat Commun 11, 332, doi:10.1038/s41467-019-14029-w (2020).

32 Paul, S. et al. Ihha induces hybrid cartilage-bone cells during zebrafish jawbone regeneration. Development 143, 2066-2076, doi:10.1242/dev.131292 (2016).

33 Barske, L. et al. Evolution of vertebrate gill covers via shifts in an ancient Pou3f3 enhancer. Proc Natl Acad Sci U S A 117, 24876-24884, doi:10.1073/pnas.2011531117 (2020).

34 Bessa, J. et al. meis1 regulates cyclin D1 and c-myc expression, and controls the proliferation of the multipotent cells in the early developing zebrafish eye. Development 135, 799-803, doi:10.1242/dev.011932 (2008).

35 Crump, J. G., Swartz, M. E., Eberhart, J. K. \& Kimmel, C. B. Moz-dependent Hox expression controls segment-specific fate maps of skeletal precursors in the face. Development 133, 2661-2669, doi:10.1242/dev.02435 (2006).

36 Hong, H. K., Lass, J. H. \& Chakravarti, A. Pleiotropic skeletal and ocular phenotypes of the mouse mutation congenital hydrocephalus (ch/Mf1) arise from a winged helix/forkhead transcriptionfactor gene. Human molecular genetics 8, 625-637 (1999).

$37 \mathrm{Xu}, \mathrm{P}$. et al. Fox proteins are modular competency factors for facial cartilage and tooth specification. Development 145, doi:10.1242/dev.165498 (2018).

38 Wei, G. et al. Ets1 and Ets2 are required for endothelial cell survival during embryonic angiogenesis. Blood 114, 1123-1130, doi:10.1182/blood-2009-03-211391 (2009).

39 Zhao, B. et al. Interferon regulatory factor-8 regulates bone metabolism by suppressing osteoclastogenesis. Nat Med 15, 1066-1071, doi:10.1038/nm.2007 (2009).

40 Sheehan-Rooney, K., Swartz, M. E., Zhao, F., Liu, D. \& Eberhart, J. K. Ahsa1 and Hsp90 activity confers more severe craniofacial phenotypes in a zebrafish model of 
654

655

656

657

658

659

660

661

662

663

664

665

666

667

668

669

670

671

672

673

674

675

676

677

678

679

680

681

hypoparathyroidism, sensorineural deafness and renal dysplasia (HDR). Dis Model Mech 6 , 1285-1291, doi:10.1242/dmm.011965 (2013).

41 Muhl, L. et al. Single-cell analysis uncovers fibroblast heterogeneity and criteria for fibroblast and mural cell identification and discrimination. Nat Commun 11, 3953, doi:10.1038/s41467020-17740-1 (2020).

42 Gerber, T. et al. Single-cell analysis uncovers convergence of cell identities during axolotl limb regeneration. Science 362, doi:10.1126/science.aaq0681 (2018).

43 Malpel, S., Mendelsohn, C. \& Cardoso, W. V. Regulation of retinoic acid signaling during lung morphogenesis. Development 127, 3057-3067 (2000).

44 Kobayashi, I. et al. Jam1a-Jam2a interactions regulate haematopoietic stem cell fate through Notch signalling. Nature 512, 319-323, doi:10.1038/nature13623 (2014).

45 Kimura, Y., Hisano, Y., Kawahara, A. \& Higashijima, S. Efficient generation of knock-in transgenic zebrafish carrying reporter/driver genes by CRISPR/Cas9-mediated genome engineering. Sci Rep 4, 6545, doi:10.1038/srep06545 (2014).

46 Thomas, E. D. \& Raible, D. W. Distinct progenitor populations mediate regeneration in the zebrafish lateral line. Elife 8, doi:10.7554/eLife.43736 (2019).

47 Fornes, O. et al. JASPAR 2020: update of the open-access database of transcription factor binding profiles. Nucleic Acids Res 48, D87-D92, doi:10.1093/nar/gkz1001 (2020).

48 Schep, A. N., Wu, B., Buenrostro, J. D. \& Greenleaf, W. J. chromVAR: inferring transcriptionfactor-associated accessibility from single-cell epigenomic data. Nat Methods 14, 975-978, doi:10.1038/nmeth.4401 (2017).

49 Brionne, A., Juanchich, A. \& Hennequet-Antier, C. ViSEAGO: a Bioconductor package for clustering biological functions using Gene Ontology and semantic similarity. BioData Min 12, 16, doi:10.1186/s13040-019-0204-1 (2019).

50 Wingender, E., Dietze, P., Karas, H. \& Knuppel, R. TRANSFAC: a database on transcription factors and their DNA binding sites. Nucleic Acids Res 24, 238-241, doi:10.1093/nar/24.1.238 (1996). 
a

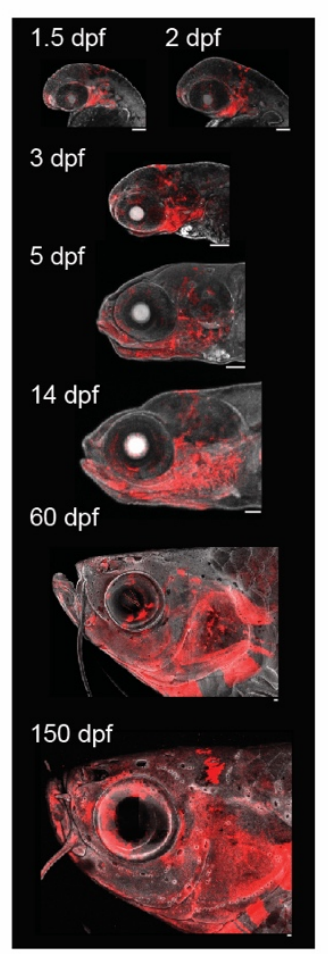

e
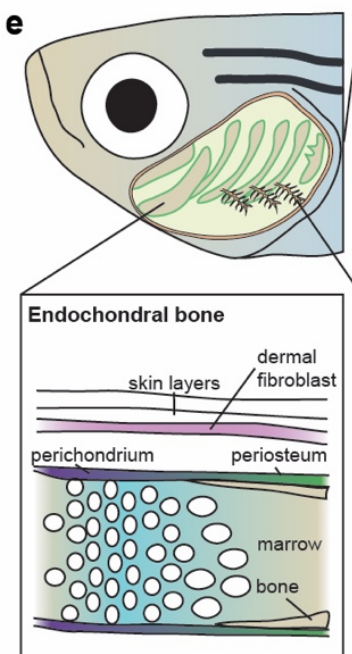

b

Head Dissection \& Dissociation
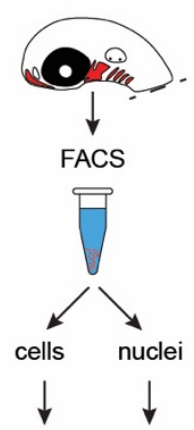

10x Genomics
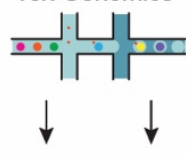

Sequencing

scRNAseq snATACseq
C 150 dpf ScRNAseq

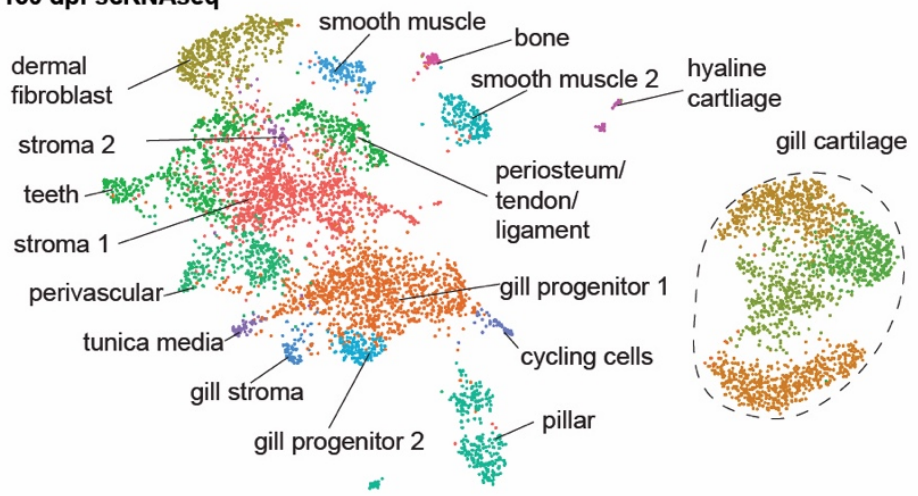

d

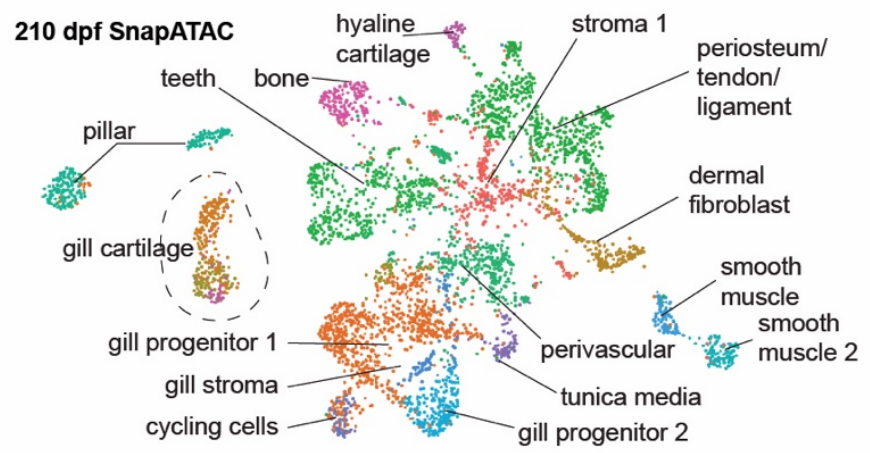

f

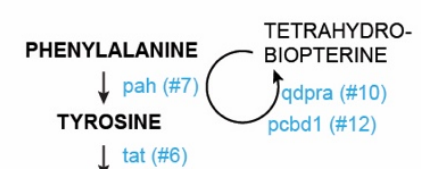

$$
\downarrow \text { tat (\#6) }
$$

4-HYDROXY

PHENYLPYRUVATE

$\downarrow$ hpdb (\#1)

HOMOGENTISATE

$\downarrow$ hgd (\#8)

4-MALEYLACETOCAETATE

$\downarrow$ gstz1(\#20)

4-FUMARYLACETOACETATE

$\swarrow$ fah $\searrow$

FUMARATE ACETOACETATE

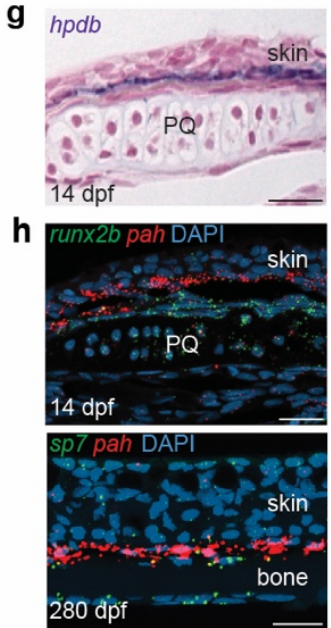

Figure 1. Single-cell transcriptomes and chromatin accessibility of CNCCs across the zebrafish lifetime. a, Sox10:Cre; actab2:IoxP-BFP-STOP-loxP-dsRed labeling of CNCCs (red) in zebrafish heads across 7 stages. b, Scheme of cell or nuclei dissociation, fluorescence activated cell sorting (FACS), and processing on the 10X Genomics platform for sequencing. c,d,

687 UMAPs of scRNAseq and SnapATAC datasets at adult stages. e, Diagram of an adult zebrafish 
689 for Phe and Tyr breakdown. 7 of 8 genes encoding catabolic enzymes are in the top 20 selectively

690 enriched genes for the dermal fibroblast cluster (numbers show rank). g, Section colorimetric in

691 situ hybridization for hpdb RNA shows expression in the dermis between the skin and

692 palatoquadrate (PQ) endochondral bone. $\mathbf{h}$, Section RNAscope in situ hybridizations show pah

693 expression in dermal fibroblasts between the runx $2 b+$ periosteum of $P Q$ and skin at $14 \mathrm{dpf}$, and

694 between sp7+ osteoblasts of intramembranous bone and skin at $280 \mathrm{dpf}$. DAPI labels nuclei in

695 blue. Scale bars, $100 \mu \mathrm{m}(\mathrm{a}), 20 \mu \mathrm{m}(\mathrm{g}, \mathrm{h})$.
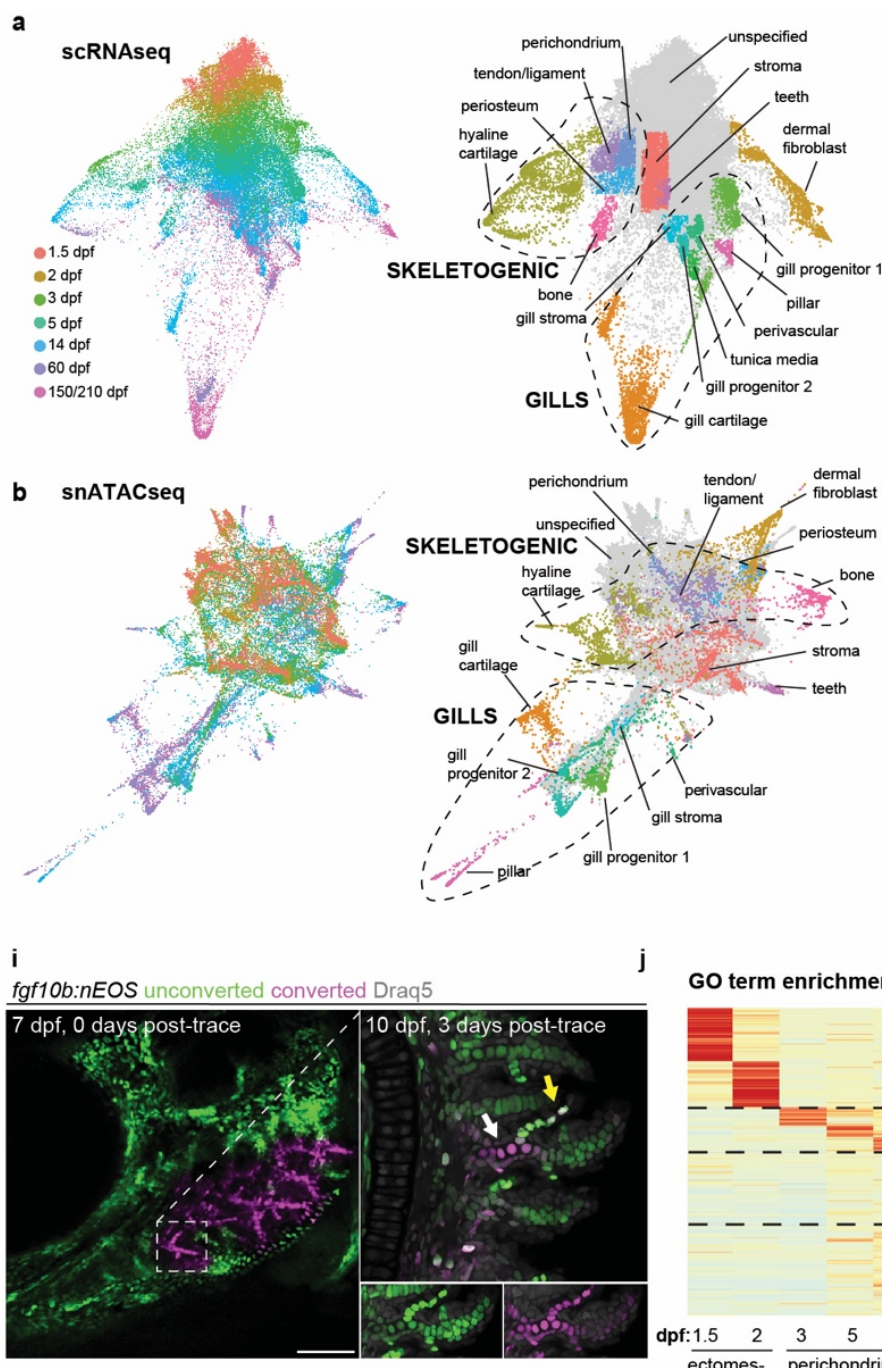

697

j

\begin{abstract}
Go term enrichment
\end{abstract}

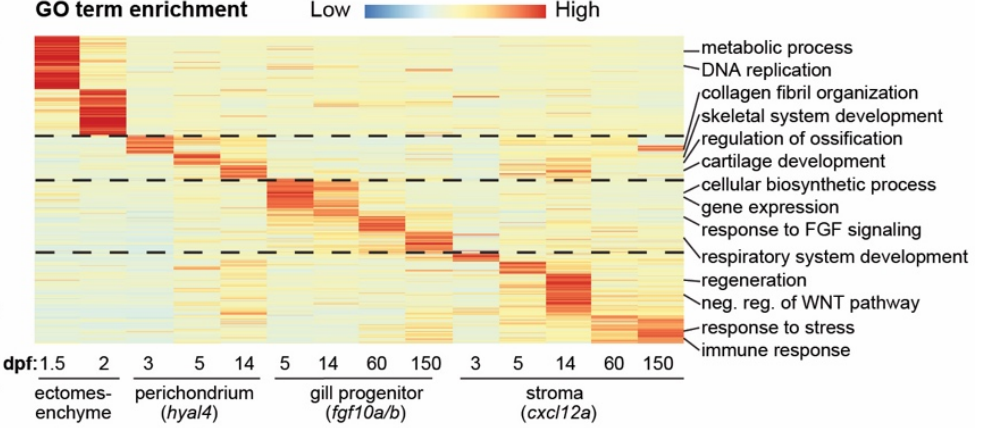

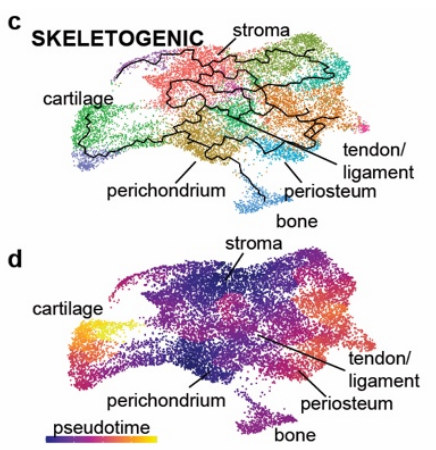
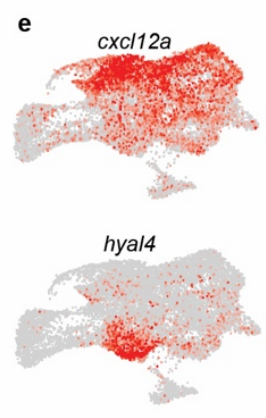

f

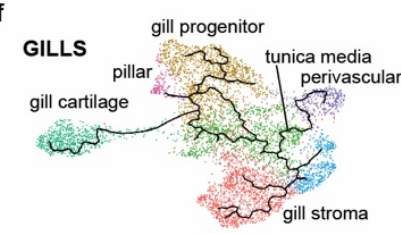

g
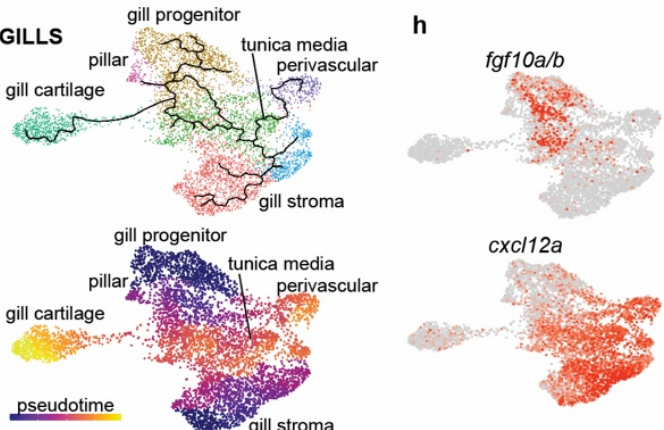

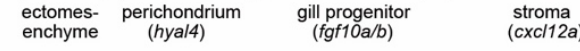

Figure 2. Progressive emergence of region-specific lineage programs. a,b, STITCH plots 
connect individual scRNAseq and snATACseq datasets across the zebrafish lifetime. Cell type annotations show divergence of CNCC ectomesenchyme into skeletogenic, gill, dermal fibroblast,

701 and stromal branches. c-e, Pseudotime analysis using Monocle3 of the skeletogenic subset

702 (combined 5 and $14 \mathrm{dpf}$ ) shows a cxcl12a+ stromal branch, and a hyal4+ branch connected to 703 cartilage, bone, periosteum, and tendon and ligament. f-h, Pseudotime analysis of gill subsets

704 (combined 5 and $14 \mathrm{dpf}$ ) shows a cxc/12a+ stromal branch, and a fgf10a/b+ branch connected to 705 gill pillar, tunica media, and perivascular cells, as well as a distinct type of gill cartilage. i, Following 706 UV-mediated photoconversion of fgf10b:nEOS from green to magenta in a subset of filaments, 707 re-imaging 3 days later revealed contribution of converted cells to pillar cells (yellow arrow, white 708 reflects mixture of converted magenta and new unconverted green fgf10b:nEOS) and gill 709 chondrocytes (white arrow) ( $n=3$ ). Draq5 labels nuclei in grey. j, Gene Ontology (GO) terms of 710 biological processes for the respective clusters at the stages indicated. Heatmap reflects the 711 negative log of the adjusted $p$ value. Ectomesenchyme is defined as the average of all cells at 7121.5 and 2 dpf stages. Scale bar, $100 \mu \mathrm{m}$. 
a

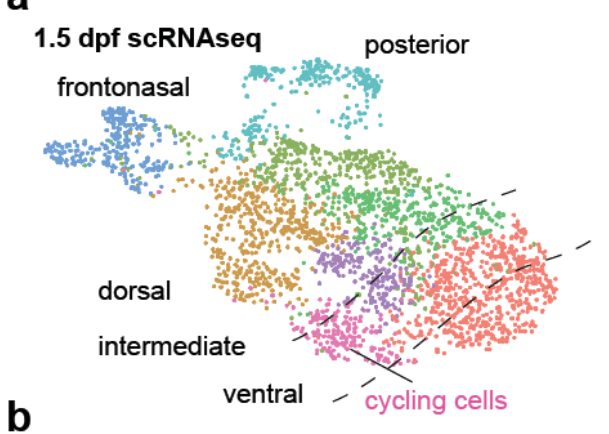

b

$1.5 \mathrm{dpf}$ SnapATAC

frontonasal $1^{\text {st }}$ arch $2^{\text {nd }} \operatorname{arch} 3^{\text {rd }}-6^{\text {th }} \operatorname{arch} 7^{\text {th }} \operatorname{arch}$

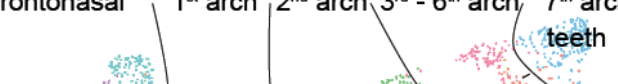

opercle

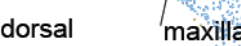

intermediate

$----$

ventral

Cnriched Motifs

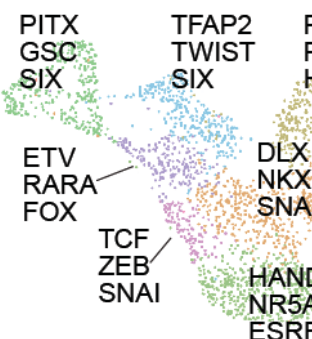

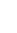

HAND

GLI

SMAD
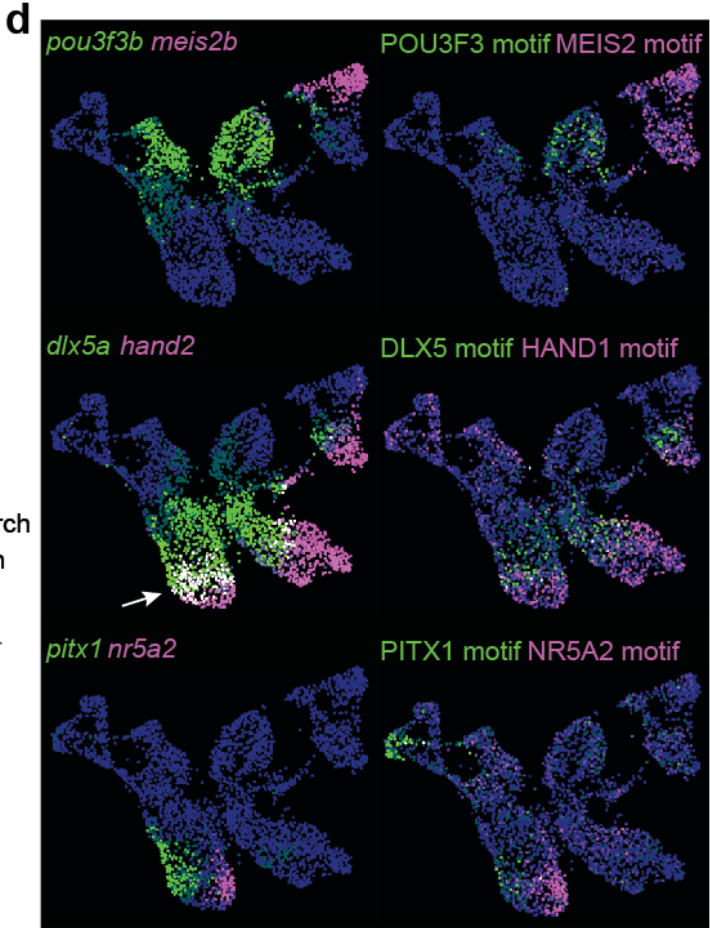

e nr5a2 sox 10:dsRed

2

3

5

eye 1
714

Figure 3. Highly resolved embryonic spatial expression domains from integrated datasets.

a,b, UMAPs at $1.5 \mathrm{dpf}$ generated by scRNAseq versus integration of scRNAseq and snATACseq datasets using SnapATAC. SnapATAC outperforms scRNAseq in resolving dorsoventral (vertical), anterior-posterior (horizontal), and major arch landmarks including a previously unappreciated oral-aboral axis in zebrafish. c, Select enriched transcription factor binding motifs for each cluster. d, Gene body activities for transcription factors and their corresponding DNAbinding motifs reveal tight correspondence with published expression patterns, including zebrafish-specific overlap of $d l \times 5 a$ and hand2 in the mandibular arch (arrow). e, Fluorescent in situ hybridization shows restricted expression of $n r 5 a 2$ in the aboral domain of the mandibular arch as predicted by SnapATAC. sox10:dsRed labels CNCCs of the arches (numbered, antidsRed antibody stain). Scale bar, $20 \mu \mathrm{m}$. 

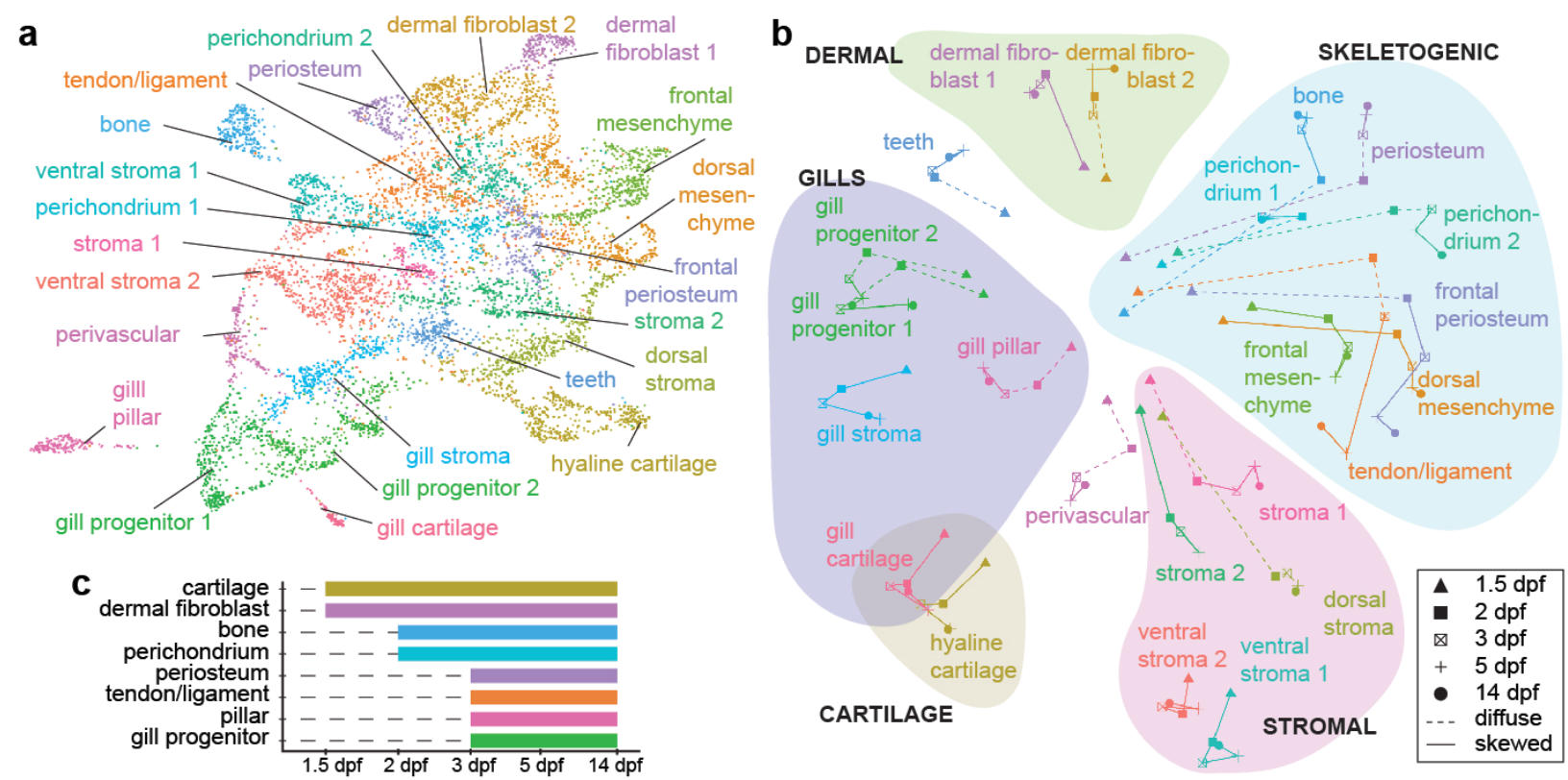

Figure 4. Cell type competency mapping through retrograde chromatin accessibility

analysis. a, UMAP of $14 \mathrm{dpf}$ SnapATAC data shows cell clusters for which top accessible peak modules were calculated for Constellations analysis. b, Constellations analysis involves mapping of cluster-specific chromatin accessibility from $14 \mathrm{dpf}$ back to earlier stages and then plotting relatedness of mapped accessibility in two dimensions. Diffuse refers to a stage where clusterspecific chromatin accessibility does not map to a discrete portion of UMAP space, and skewed where it does (see Methods for details). Groups of related cell types are color-coded. c, Graphical representation from the Constellations analysis of when chromatin accessibility of major cell types first shows a skewed distribution in UMAP space, suggestive of establishment of competency. a, UMAP of $14 \mathrm{dpf}$ SnapATAC data shows cell clusters for which top accessible peak modules were calculated for Constellations analysis. b, Constellations analysis involves mapping of clusterspecific chromatin accessibility from $14 \mathrm{dpf}$ back to earlier stages and then plotting relatedness

737 of mapped accessibility in two dimensions. Diffuse refers to a stage where cluster-specific 738 chromatin accessibility does not map to a discrete portion of the UMAP space, and skewed to 739 where it does (see Methods for details). Groups of related cell types are color-coded. c, Graphical 740 representation from the Constellations analysis of when chromatin accessibility of major cell types 741 first shows a skewed distribution in UMAP space, suggestive of establishment of competency. 


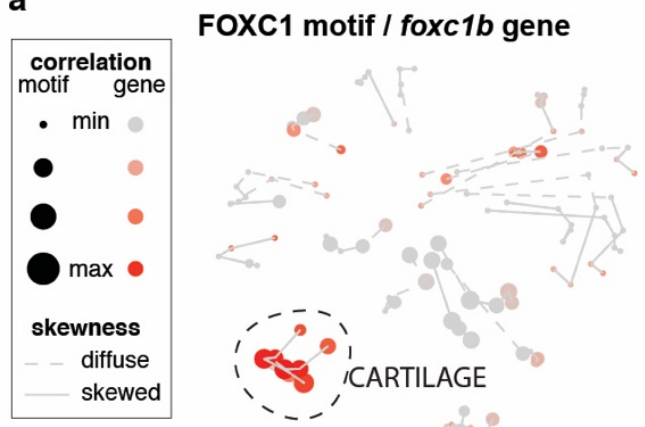

\section{LEF1 motif / lef1 gene}

-

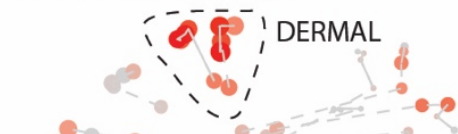

GATA3 motif / gata3 gene

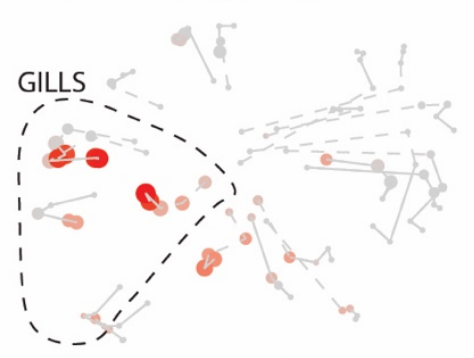

ETS2 motif / ets2 gene

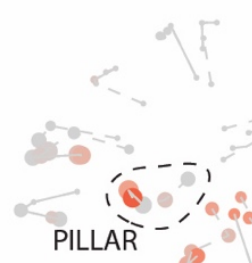

b

cartilage peaks

dermal fibroblast 1 peaks

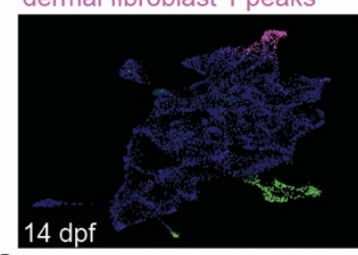

$\mathbf{c}_{\text {fox }}$

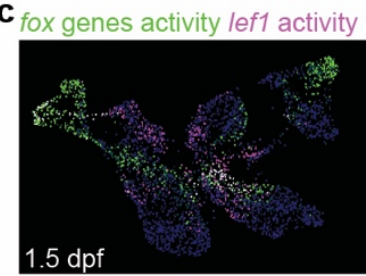

cartilage peaks dermal fibroblast 1 peaks

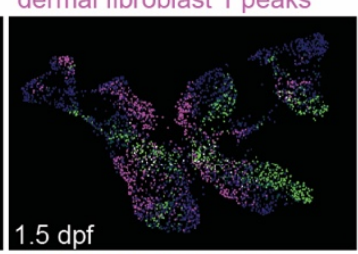

FOX motifs LEF1 motif

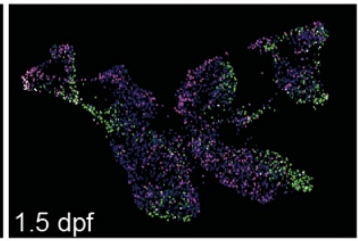

IRF8 motif / irf8 gene

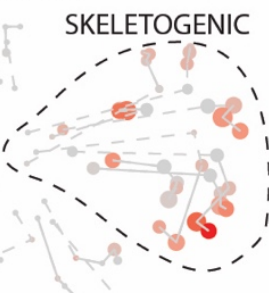

d

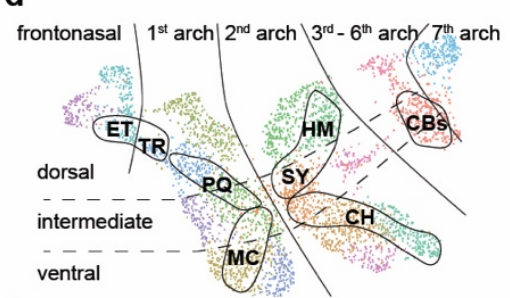

e

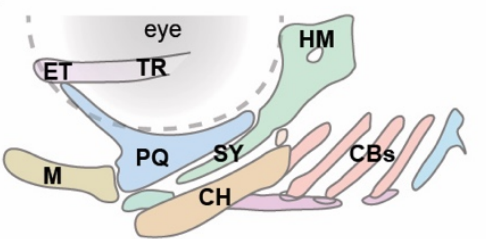

CEBPA motif / cebpa gene

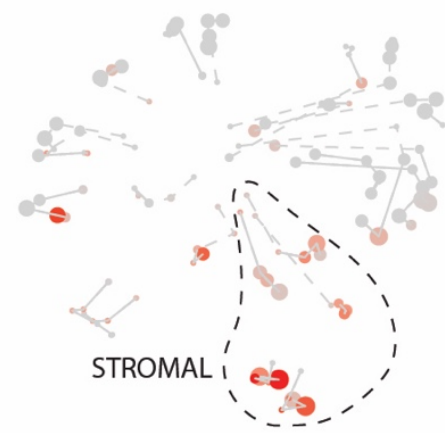

f

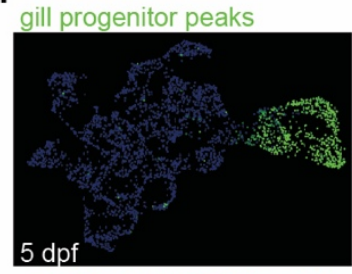

gata3 activity GATA3 motif

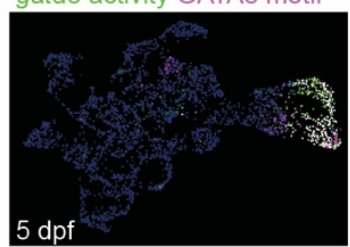

Figure 5. Constellations analysis reveals candidate transcription factors for lineage

744 priming. a, Mapping onto the Constellations plot of transcription factors with correlated gene body

745 activity and DNA-binding motif enrichment in specific clusters. Sizes of circles denote correlation

746 of peak module mapping to motifs, and red color to gene body activities. b, Top peak modules

747 for hyaline cartilage and dermal fibroblast 1 clusters at $14 \mathrm{dpf}$ mapped onto $14 \mathrm{dpf}$ and $1.5 \mathrm{dpf}$

748 SnapATAC UMAPs. c, Summed gene body activities of foxc1a, foxc $1 b$, foxf1, foxf2a, foxf $2 b$ and

749 summed FOXC1, FOXF1, and FOXF2 motifs at 1.5 dpf correlate with cartilage peak mapping, 
752 cartilaginous elements of the week-old skeleton: ceratobranchials (CBs), ceratohyal $(\mathrm{CH})$,

753 ethmoid (ET), hyomandibula (HM), Meckel's (M), palatoquadrate (PQ), symplectic (SY), and

754 trabecula (TR). f, Mapping of the top peak module for $14 \mathrm{dpf}$ gill progenitor clusters onto $5 \mathrm{dpf}$

755 SnapATAC UMAP shows correlation with gata3 gene body activity and GATA3 motif.

a

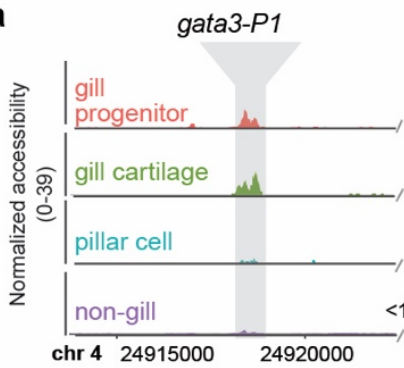

C

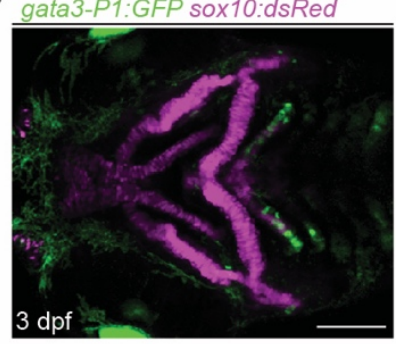

f

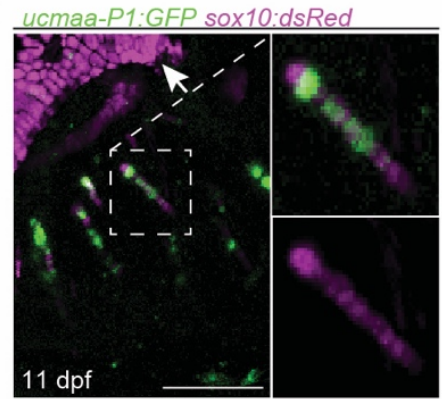

d
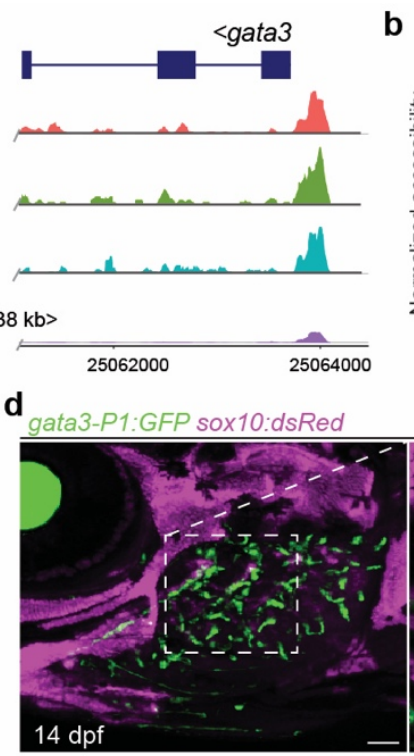

g

Model for gill-specific program $1.5 \mathrm{dpf} \quad 14 \mathrm{dpf}$

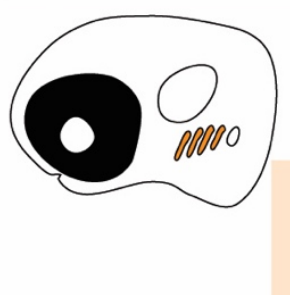

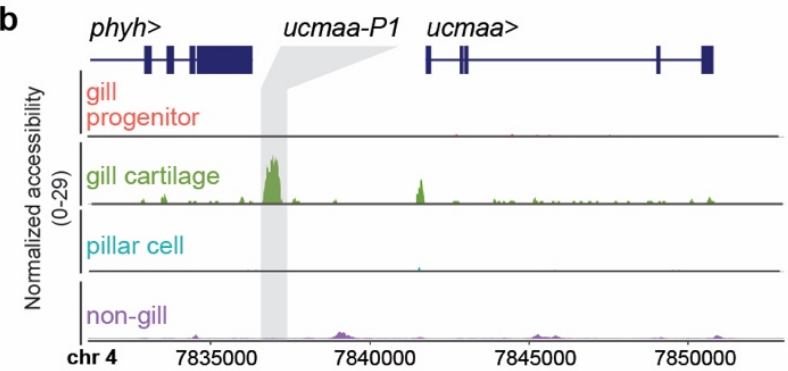

e
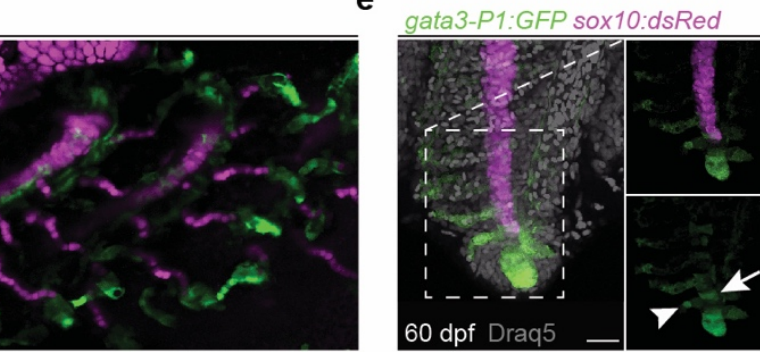

gill cartilage
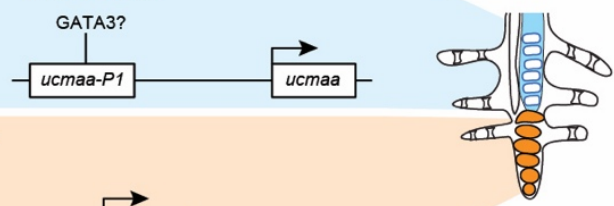

Figure 6. Gata3 activity distinguishes the gill-specific lineage. a,b, Genome tracks show chromatin accessibility for cell clusters from snATACseq data at $14 \mathrm{dpf}$. Grey shading shows gillspecific accessible regions near the gata3 and ucmaa genes. Chromosome positions refer to the

761 GRCz11 genome assembly. c-f, gata3-P1:GFP drives expression in the posterior gill-forming 
762 arches at $3 \mathrm{dpf}$, the gill filament system at $14 \mathrm{dpf}$, and gill progenitors at the tips of primary

763 filaments at $60 \mathrm{dpf}$, as well as some pillar cells (arrowhead) and gill chondrocytes (arrow) near

764 the growing tips. sox10:dsRed labels cartilage and Draq5 nuclei. f, ucmaa-P1:GFP drives highly

765 restricted expression in sox10:dsRed+ gill chondrocytes (boxed region shown in merged and

766 single channels to the right) but not hyaline cartilage (top left). $\mathbf{g}$, Model shows initiation of gata3

767 expression in the posterior gill-forming arches through the gata3-P1 enhancer, maintenance of

768 gata3 in gill progenitors at the tips of growing filaments, and expression of ucmaa in gill cartilage

769 through the ucmaa-P1 enhancer. Both gata3-P1 and ucmaa-P1 contain predicted Gata3 binding

770 sites. Scale bars, $100 \mu \mathrm{m}(\mathrm{c}, \mathrm{d}, \mathrm{f}), 20 \mu \mathrm{m}(\mathrm{e})$. 